\title{
Impulsive SEP Events (and Flares)
}

\begin{abstract}
${ }^{3} \mathrm{He}$-rich, Fe-rich, and enriched in elements with $\mathrm{Z}>50$, the abundances of solar energetic particles (SEPs) from the small impulsive SEP events stand out as luminaries in our study. The ${ }^{3} \mathrm{He}$ is enhanced by resonant wave-particle interactions. Element abundances increase 1000-fold as the 3.6 power of the mass-to-charge ratio $A / Q$ from He to heavy elements like $\mathrm{Au}$ or $\mathrm{Pb}$, enhanced during acceleration in islands of magnetic reconnection in solar jets, and probably also in flares. This power-law of enhancement vs. $A / Q$ implies $Q$ determined by a source temperature of 2.5-3.2 MK, typical of jets from solar active regions where these impulsive SEPs occur. However, a few small events are unusual; several have suppressed ${ }^{4} \mathrm{He}$, and rarely, a few very small events with steep spectra have elements $\mathrm{N}$ or $\mathrm{S}$ greatly enhanced, perhaps by the same resonant-wave mechanism that enhances ${ }^{3} \mathrm{He}$. Which mechanism will dominate? The impulsive SEP events we see are associated with narrow CMEs, from solar jets where magnetic reconnection on open field lines gives energetic particles and CMEs direct access to space. Gamma-ray lines tell us that the same acceleration physics may occur in flares.
\end{abstract}

Impulsive SEP events were first identified by their unusual enhancements of ${ }^{3} \mathrm{He} /{ }^{4} \mathrm{He}$, with $\sim 1000$-fold increases over the abundance ${ }^{3} \mathrm{He} /{ }^{4} \mathrm{He} \approx 5 \times 10^{-4}$ in the solar wind, frequently with ${ }^{3} \mathrm{He} /{ }^{4} \mathrm{He}>1$, and occasionally with ${ }^{3} \mathrm{He} / \mathrm{H}>1$. Next we found enhancements of $\mathrm{Fe} / \mathrm{C}$ or $\mathrm{Fe} / \mathrm{O}$ of $\sim 10$, which were more-stable indicators of impulsive events, since ${ }^{3} \mathrm{He} /{ }^{4} \mathrm{He}$ varies widely. Then $\sim 1000$-fold increases in elements with $(76 \leq Z \leq 82) / O$ were added to the unusual picture.

Despite the huge enhancements of ${ }^{3} \mathrm{He}$, the isotopes ${ }^{2} \mathrm{H}$ and ${ }^{3} \mathrm{H}$ are not observed in SEPs $\left(<1 \%\right.$ of ${ }^{3} \mathrm{He}$ according to Serlemitsos and Balasubrahmanyan 1975). Observations of $\gamma$-ray lines and neutrons show the presence of nuclear reactions in the low corona during flares (e.g. Ramaty and Murphy 1987), but isotopes of Li, Be, 
and $\mathrm{B}$ have never been observed in SEPs. Limits on $\mathrm{Be} / \mathrm{O}$ or $\mathrm{B} / \mathrm{O}$ in large SEP events are $<4 \times 10^{-4}$ (e.g. Cook et al. 1984). Reaction secondaries are trapped on flare loops and cannot escape, and the ${ }^{3} \mathrm{He}$ we see is not a nuclear-reaction product, it is enhanced by resonant wave-particle reactions (e.g. Temerin and Roth 1992; see Sect. 2.5.2). In fact, as we have seen, only particles accelerated on open field lines, e.g. in solar jets (or at shock waves), can ever escape.

\subsection{Selecting Impulsive Events}

Many years ago, Reames (1988) examined the distribution of all daily abundance averages with measurable $\mathrm{Fe} / \mathrm{O}$ ratios during 8.5 years, and found a bimodal distribution with peaks near $\mathrm{Fe} / \mathrm{O} \approx 0.1$ and $\mathrm{Fe} / \mathrm{O} \approx 1.0$. The technique was free from bias related to individual event selection, although long-duration events were certainly more heavily sampled. Periods with Fe/O near 0.1 had unremarkable abundances of other elements, but those near 1.0 also had enhancements in ${ }^{3} \mathrm{He} /{ }^{4} \mathrm{He},{ }^{4} \mathrm{He} / \mathrm{H}$, and e/p ratios. While the two distributions of $\mathrm{Fe} / \mathrm{O}$ did have an overlap region, largely because the poor statistics available at that time spread the distributions, the results showed that $\mathrm{Fe} / \mathrm{O}$ at about 2-5 $\mathrm{MeV} \mathrm{amu}^{-1}$ was more reliable for selecting candidate periods (e.g. Fig. 4.1) for impulsive SEP events than ${ }^{3} \mathrm{He} /{ }^{4} \mathrm{He}$, which depends strongly upon energy (e.g. Fig. 4.4).

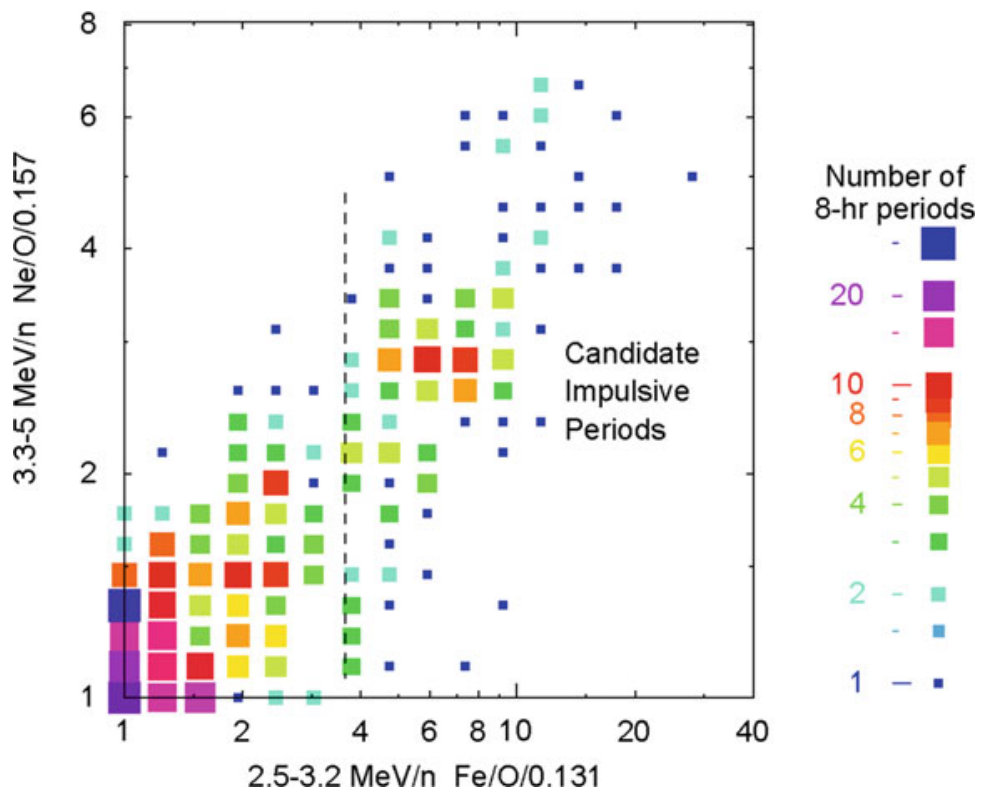

Fig. 4.1 Measured relative enhancements in $\mathrm{Ne} / \mathrm{O}$ vs. $\mathrm{Fe} / \mathrm{O}$ for 8 -h periods during 19 years are binned for all periods with errors of $20 \%$ or less (Reames et al. 2014a (C) Springer) 
A more-recent version of the bimodal abundance study is the two-dimensional histogram shown in Fig. 4.1, based upon much more accurate data. Here 8-h measurements of $\mathrm{Ne} / \mathrm{O}$ vs. Fe/O are binned for a 19-year period, and this time we have the luxury of requiring $20 \%$ accuracy to prevent excessive spreading of the distributions. Of course, it is still true that gradual events occupy many more 8-h periods and impulsive events, with lower intensities, are less likely to achieve $20 \%$ accuracy, but the presence of two peaks is clear.

Time periods near coordinates $(1,1)$ in Fig. 4.1, occur during large gradual SEP events for which the normalization was chosen. The peak near $(6,3)$ in the figure represents impulsive events, but the $\mathrm{Ne} / \mathrm{O}$ value was not actually used for selection of candidate periods for defining impulsive SEP events.

\subsection{Sample Impulsive Events}

Figure 4.2 shows intensities of several particle species in a sample of impulsive SEP events with various properties. In events 1,2 , and 5 , we have ${ }^{3} \mathrm{He} /{ }^{4} \mathrm{He}>>1$, and in event $1,{ }^{3} \mathrm{He}>\mathrm{H}$. In events 1 and 2 the $\mathrm{O}$, which may seem high relative to $\mathrm{Fe}$, is actually background from anomalous cosmic-ray $\mathrm{O}$ and is present at almost the same rate before and after the events. In events 5 and $6, \mathrm{O}$ is closer to ${ }^{4} \mathrm{He}$ than in other events, these "He-poor" events have low ${ }^{4} \mathrm{He} / \mathrm{O}$. Events $6-13$ on the bottom row are an order of magnitude larger in ${ }^{4} \mathrm{He}, \mathrm{O}$, or Fe than those in the first row, and heavy elements begin to appear; these larger events are also not as strongly ${ }^{3} \mathrm{He}$-rich. Instrument limitations: only groups of elements are resolved above $Z=34$ and, when ${ }^{3} \mathrm{He} /{ }^{4} \mathrm{He}<0.1,{ }^{3} \mathrm{He}$ is poorly resolved and is not plotted.

\subsection{Energy Dependence}

Some sample energy spectra in ${ }^{3}$ He-rich events are shown in Fig. 4.3.

The spectra on the left in Fig. 4.3 appear as broken power-law spectra while those on the right are more curved and show large energy variations in ${ }^{3} \mathrm{He} /{ }^{4} \mathrm{He}$ as seen in Fig. 4.4. Abundance ratios of $\mathrm{Fe} / \mathrm{O}$ show much less spectral variation.

The ${ }^{3} \mathrm{He} /{ }^{4} \mathrm{He}$ variations shown in Fig. 4.4 make it difficult to characterize an event by this ratio, which seems to peak in the region of $1-10 \mathrm{MeV} \mathrm{amu}^{-1}$. Fe/O at a few $\mathrm{MeV} \mathrm{amu}^{-1}$ is a better-behaved alternative for defining impulsive events, as suggested in Fig. 4.1.

Liu et al. (2006) have been able to fit the complex spectra of ${ }^{3} \mathrm{He}$ and ${ }^{4} \mathrm{He}$ with a model of stochastic acceleration by a power-law spectrum of plasma-wave turbulence, presumably associated with magnetic reconnection. This work follows the tradition of stochastic acceleration involving the general transfer of energy from waves to particles (see reviews: Miller et al. 1997; Miller 1998). These models have difficulty explaining the strong $A / Q$-dependent enhancements extending to heavy elements that we will discuss in Sects. 4.5 and 4.6. 

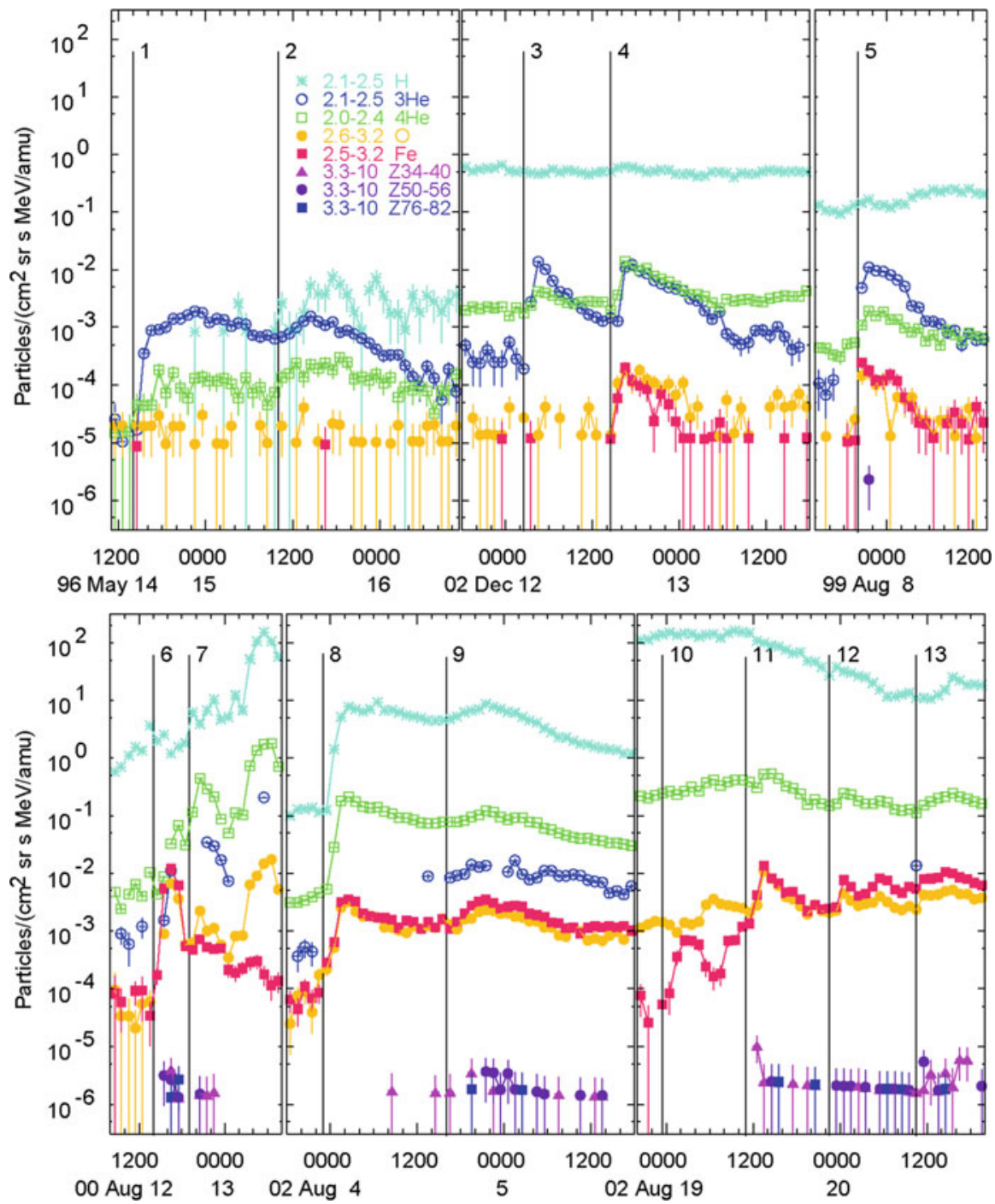

Fig. 4.2 Intensities of $\mathrm{H},{ }^{3} \mathrm{He},{ }^{4} \mathrm{He}, \mathrm{O}, \mathrm{Fe}$, and heavy elements are shown as a function of time during 13 impulsive SEP events (see text, Reames and Ng 2004 (C) AAS)

\subsection{Abundances for $Z \leq \mathbf{2 6}$}

Given the spectra and variations of ${ }^{3} \mathrm{He}$ we have seen, it is not surprising that the ${ }^{3} \mathrm{He} /{ }^{4} \mathrm{He}$ ratio is uncorrelated with other abundance ratios as seen in Fig. 4.5. This was known to Mason et al. (1986) and Reames et al. (1994) and is often taken as 

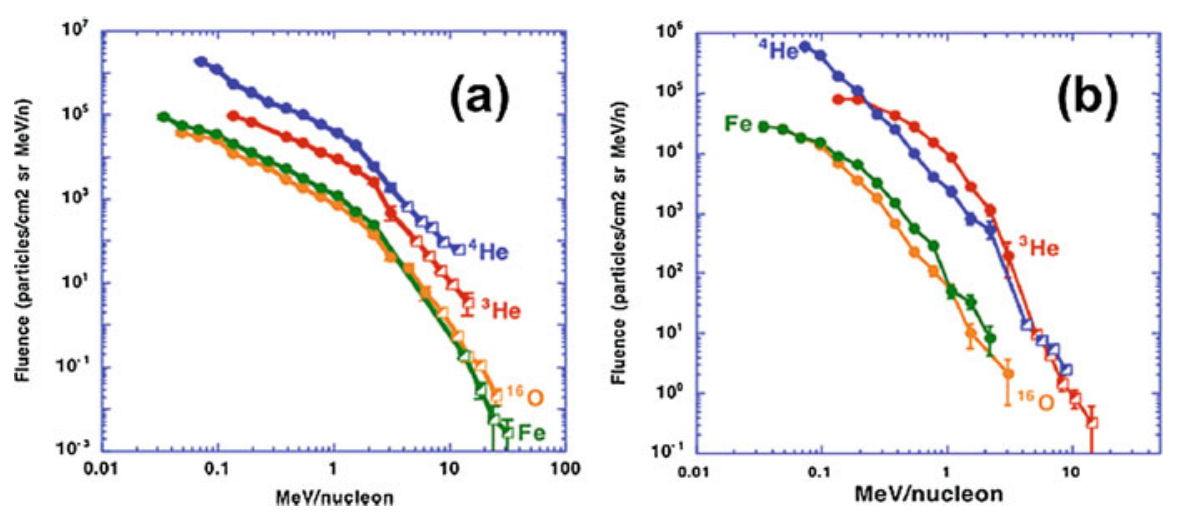

Fig. 4.3 Spectra of ${ }^{3} \mathrm{He},{ }^{4} \mathrm{He}, \mathrm{O}$ and $\mathrm{Fe}$ are shown in the (a) 9 September 1998 and the (b) 21 March 1999 events (Mason et al. 2002b, (C) AAS; Mason 2007)

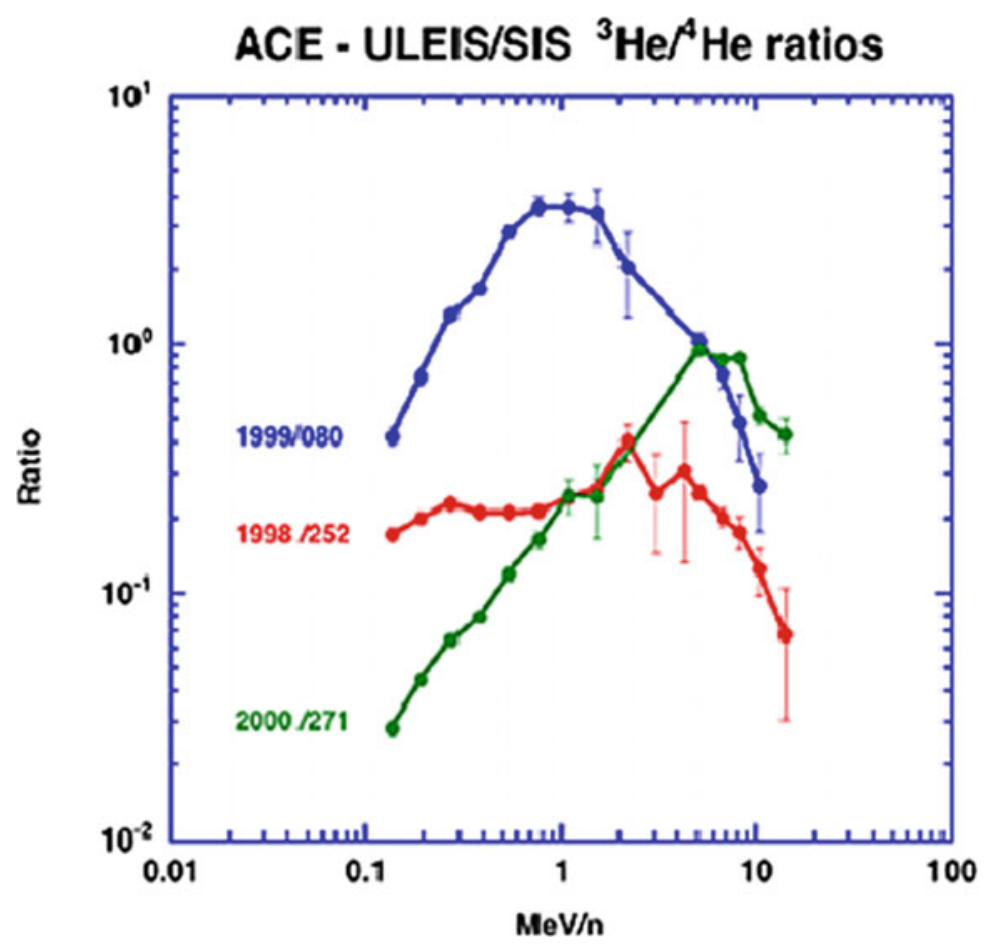

Fig. 4.4 Energy dependence is shown for ${ }^{3} \mathrm{He} /{ }^{4} \mathrm{He}$ ratios. Red and blue are for events shown in Fig. 4.3a, b, respectively. The green event is 27 September 2000 (Mason et al. 2002b (C) AAS; Mason 2007)

evidence that the mechanism of ${ }^{3} \mathrm{He}$ enhancement is different from that causing enhancement of $\mathrm{Fe} / \mathrm{O}$ and heavy elements.

The average enhancements of the elements from ${ }^{4} \mathrm{He}$ through $\mathrm{Fe}$ were summarized by Reames $(1995,1999)$ as seen in Fig. 4.6. 


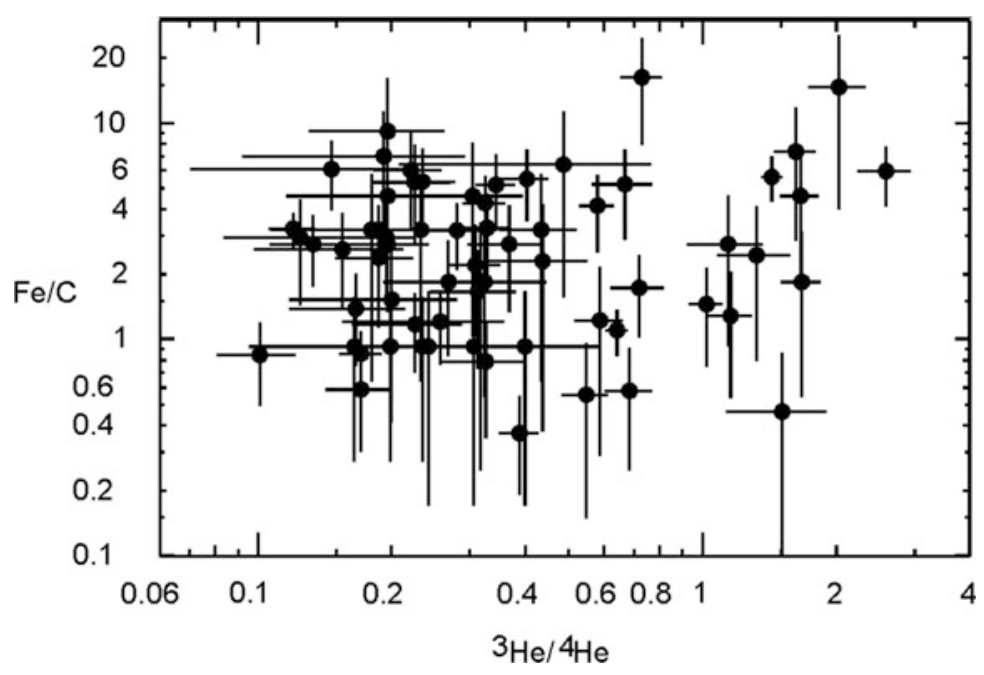

Fig. 4.5 Cross plots of $\mathrm{Fe} / \mathrm{C}$ vs. ${ }^{3} \mathrm{He} /{ }^{4} \mathrm{He}$ at $1.3-1.6 \mathrm{MeV}$ amu ${ }^{-1}$ in impulsive SEP events shows little evidence of correlation (Reames 1999; adapted from Reames et al. 1994 (C) AAS)

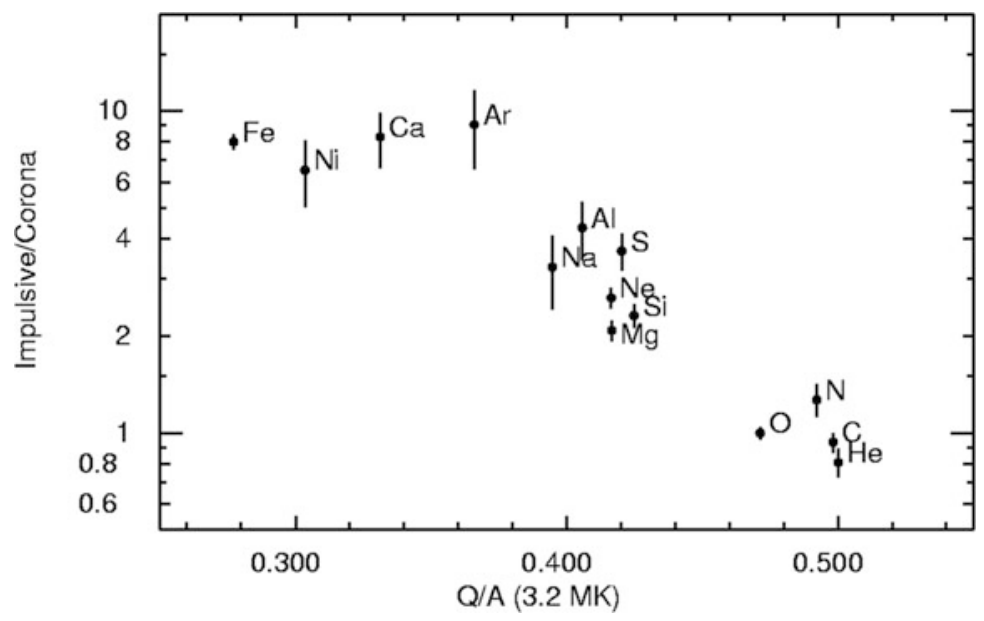

Fig. 4.6 Average abundance enhancements of elements in impulsive SEP events vs. $Q / A$ at 3.2 MK as of 1995 as shown by Reames (1999 (C) Springer)

It was suggested by Reames et al. (1994) that the grouping of the enhancements of ${ }^{4} \mathrm{He}, \mathrm{C}, \mathrm{N}$, and $\mathrm{O}$ occurs because $\mathrm{C}, \mathrm{N}$, and $\mathrm{O}$, are fully ionized, like ${ }^{4} \mathrm{He}$, and thus have $Q / A=0.5$. Ions in the group from $\mathrm{Ne}-\mathrm{S}$ have closed shells of 2 orbital electrons and $Q / A \approx 0.4$. This occurs at a temperature of about 3-5 MK, as we shall see. The observation that ${ }^{4} \mathrm{He}$ and $\mathrm{C}$ are the same supports the idea that $A / Q=2$ for both, and matter traversal is excluded since the dependence cannot be $A / Q^{2}$. 

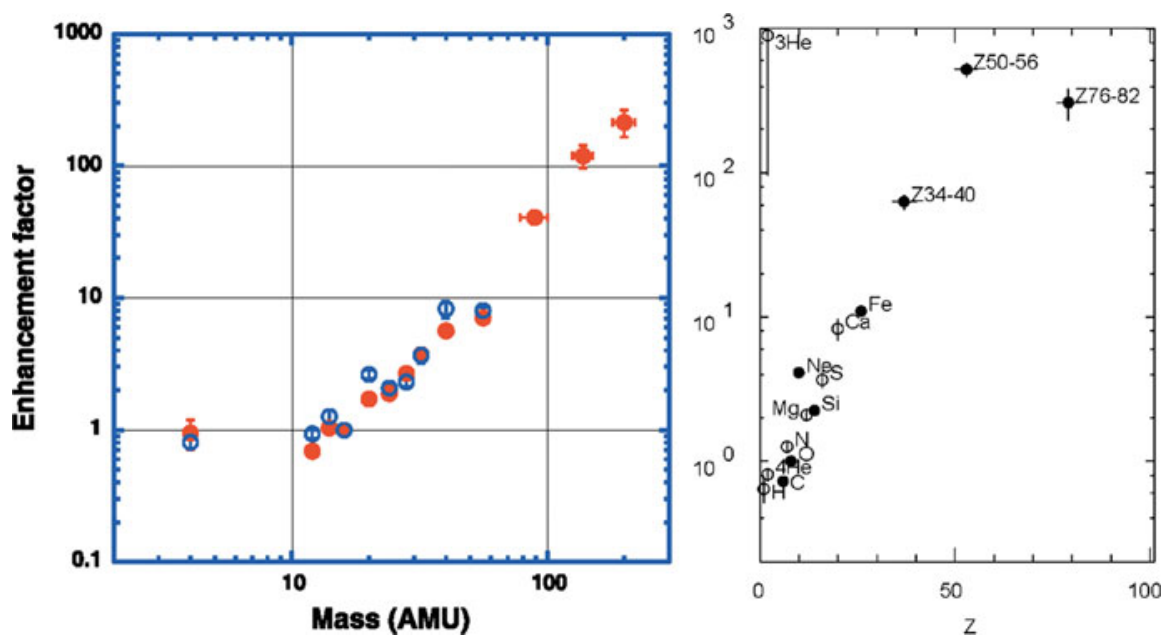

Fig. 4.7 Enhancements relative to solar system and coronal abundances are extended to high masses at $0.1-1.0 \mathrm{MeV} \mathrm{amu}{ }^{-1}$ in the left panel (red, Mason et al. 2004 (C) AAS) and to high $Z$ at 3.3-10 MeV amu ${ }^{-1}$ in the right (Reames and $\mathrm{Ng} 2004$ (C) AAS). Open symbols are from Reames (1995)

\subsection{Abundances for $34 \leq Z \leq 82$}

Beginning with the launch of the Wind spacecraft late in 1994, abundances of elements in the remainder of the periodic table well above Fe started to become available on a regular basis (Reames 2000). Although resolution of individual elements was not possible, the pattern of enhancement of element groups gave a new perspective to the term "enhancement" as the high- $Z$ elements approached 1000 -fold enhancements, comparable with those of ${ }^{3} \mathrm{He}$. Subsequently, two completely different instrument techniques yielded: (1) the abundances vs. $A$ at $0.1-1.0 \mathrm{MeV} \mathrm{amu}^{-1}$ up to $A=200$ (Mason et al. 2004) and (2) the abundances vs. $Z$ at 3.3-10 MeV amu ${ }^{-1}$ up to $Z \approx 82$ (Reames and $\mathrm{Ng} 2004$ ). Both are seen in Fig. 4.7.

Reference abundances used for Fig. 4.7 are solar system abundances for the red symbols in the left panel and solar system abundances corrected for FIP (see Chap. 8) to simulate coronal abundances in the right panel

\subsection{Power-Law Enhancements in A/Q: Source-Plasma Temperatures}

The atomic physics that describes ionization states as a function of plasma temperature $T$ has been studied for many years. Figure 4.8 shows $A / Q$ vs. $T$, based on $Q$ vs. $T$ from atomic physics, which was used by Reames et al. (2014a) to determine the appropriate value of $T$ for the power-law fit for impulsive SEP events shown in 

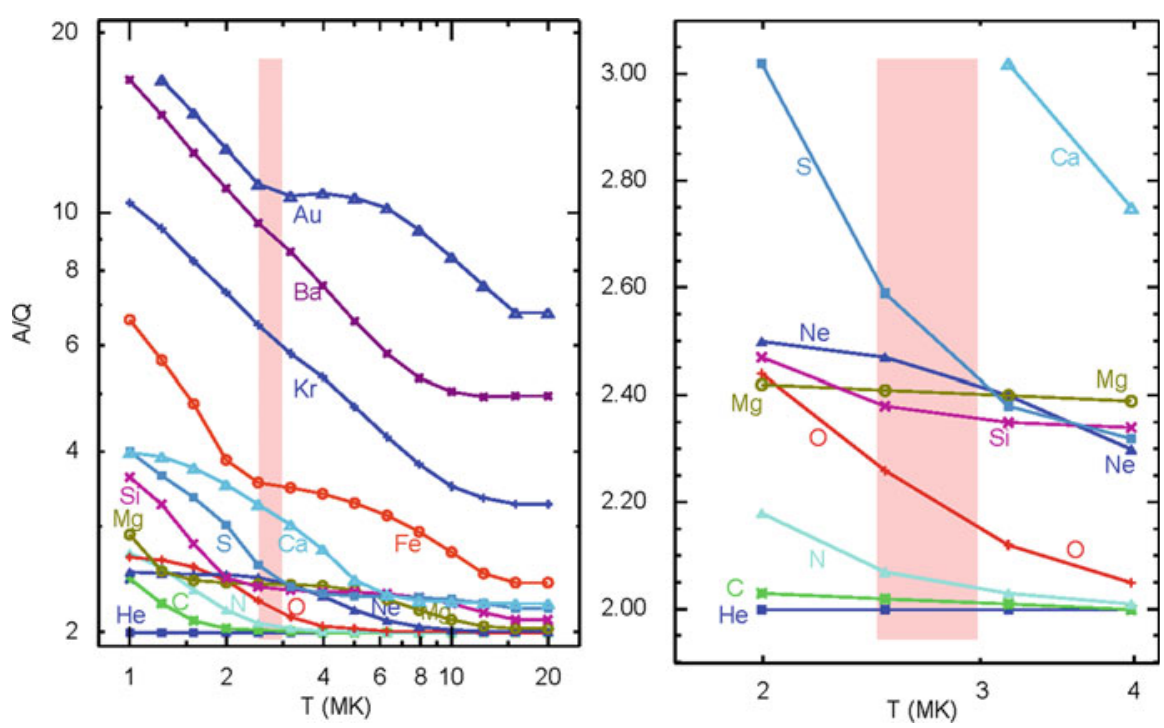

Fig. 4.8 $A / Q$ is shown as a function of equilibrium temperature for various elements (left panel) and is enlarged for low $Z$ (right panel). Data on elements below Fe are from Arnaud and Rothenflug (1985), Fe from Arnaud and Raymond (1992) and sample element data in the high- $Z$ region from Post et al. (1977). The region used for Fe-rich impulsive SEP events is shaded

Fig. 4.9. Values of $Q$ vs. $T$ below Fe are from Arnaud and Rothenflug (1985), Fe is from Arnaud and Raymond (1992) and elements in the high- $Z$ region from Post et al. (1977).

Reames et al. (2014a) found a temperature that was somewhat lower than in earlier work. They noted that $A / Q$ for $\mathrm{Ne}$ is higher than that for $\mathrm{Mg}$ or $\mathrm{Si}$ in this region $T \leq 3.0 \mathrm{MK}$ and would help explain the observation that, in the impulsive event averages, $\mathrm{Ne} / \mathrm{O}>\mathrm{Mg} / \mathrm{O}>\mathrm{Si} / \mathrm{O}$ (see right panel of Fig. 4.8). Also, $A / Q$ for $\mathrm{O}$ was beginning to approach $\approx 2.2$ in the region; this would help explain the "Hepoor" events observed with low ${ }^{4} \mathrm{He} / \mathrm{O}$. Finally, $A / Q$ values in the $2.5-3.2-\mathrm{MK}$ region fit the enhancements in the elements with $Z \geq 34$ quite well.

It is also possible to determine a best-fit temperature and a power-law fit for individual impulsive SEP events. Each impulsive event has measured enhancements for the elements and each temperature in a region of interest has its own pattern of $A$ / $Q$. We fit the enhancements vs. $A / Q$ for each temperature, note the values of $\chi^{2}$ for each fit, and then choose the fit, and temperature, with the minimum $\chi^{2}$. Values of $\chi^{2}$ vs. $T$ are shown for 111 impulsive events in Fig. 4.10. The number of events with minima at each temperature is listed along the $T$ axis. The right panel shows the spread and magnitude of the enhancements.

It is noteworthy that temperatures of 2.5-3.2 MK we find for impulsive SEP events are quite appropriate for solar jets from active regions (see Fig. 14 of Raouafi et al. 2016). The larger jets are associated with active regions. 


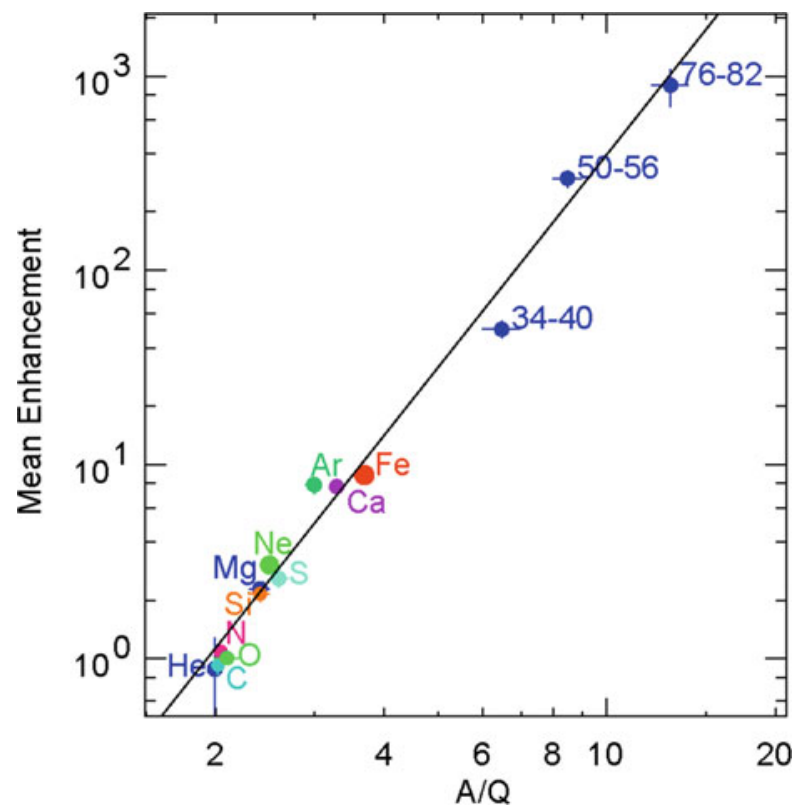

Fig. 4.9 The mean enhancement in the abundances of elements in impulsive SEP events relative to reference gradual SEP events is shown as a function of $A / Q$ of the element at $\sim 3 \mathrm{MK}$. For the leastsquares fit line shown in the figure the enhancement varies as the $3.64 \pm 0.15$ power of $A / Q$ (Reames et al. 2014a, (C) Springer)
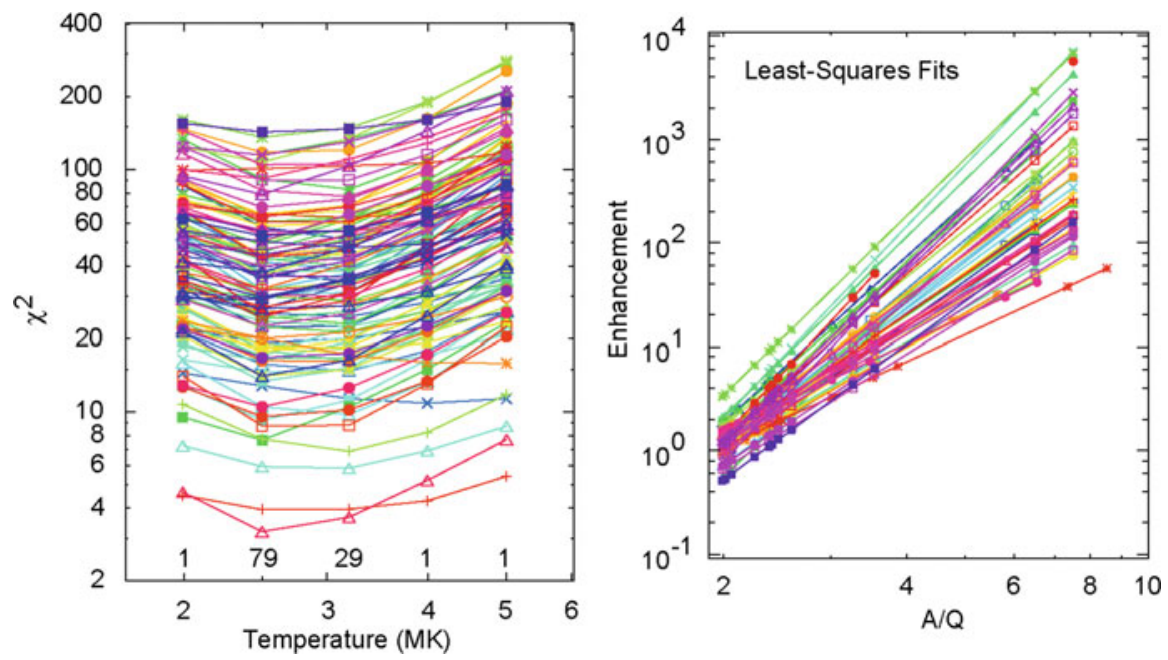

Fig. 4.10 The left panel shows $\chi^{2}$ vs. $T$ for all 111 impulsive SEP events using different colors and symbols for each event. The number of events with $\chi^{2}$ minima at each temperature is shown along the bottom of the panel. The right panel shows the distribution of the fits (Reames et al. 2014b, (C) Springer) 
Figure 4.11 shows fits of enhancement vs. $A / Q$ obtained for six individual impulsive SEP events. The event numbers listed with the onset time in each panel correspond to the numbers in the published list of Reames et al. (2014a) to provide continuity. In performing the least-squares fits for these impulsive events, errors used to determine weighting factors have been increased by $20 \%$ of the values, in quadrature with the statistical errors (Reames et al. 2014b). The physical origin of this $20 \%$ variation observed in the abundances, presumably local, is not entirely understood, but its necessity is clear.

Deviations of individual abundances of $\mathrm{Ne}, \mathrm{Mg}, \mathrm{S}$, and $\mathrm{Ca}$ above the fit lines can be seen sometimes in Fig. 4.11. In these cases, C, O, and Fe may fall below to balance the fit. In some other events larger excursions are seen, especially for Ne. These variations are not understood, but resonant enhancements at specific values of $A / Q$ are a possibility or, more likely, just local abundance variations of the solar corona. The observed variations do not appear to be variations in the FIP bias of the underlying coronal material (see Chap. 8) but may be local variations in individual element abundances.

Note that, if these ions at this energy of 3-5 MeV amu ${ }^{-1}$ had traversed a significant amount of material during or prior to acceleration, all the elements from $\mathrm{He}$ up to $\mathrm{Ne}, \mathrm{Mg}$, and $\mathrm{Si}$ would be fully ionized with $A / Q \approx 2.0$, which is not consistent with observed power-law enhancements.

For the most part, abundance variations in successive SEP events in a sequence do not seem to be correlated, as seen in the example in Fig. 4.12. However, the He abundance may be an exception.

The element He, with the highest value of FIP $=24.6 \mathrm{eV}$, may be the last element to become ionized on its transit across the solar chromosphere and into the corona. Thus its ion abundance may lag behind those of other high-FIP elements. Presumably this condition could apply throughout a region where multiple impulsive SEP events occur. However, for the events shown in Fig. 4.12, $\mathrm{He} / \mathrm{C}$ (or $\mathrm{He} / \mathrm{O}$ ) is suppressed by a factor of 5 in the earlier event and a factor of 10 in the later one. Except for coincidence, there is no known reason that the suppression of He should increase with time. However, in these "He-poor" events $\mathrm{He} / \mathrm{C}$ often decreases with energy and $\mathrm{He} / \mathrm{O}$ can be as low as $\approx 2$ at $\approx 8 \mathrm{MeV}$ amu $^{-1}$ (Reames 2019 ). ${ }^{3} \mathrm{He} /{ }^{4} \mathrm{He}$ is often high in these He-poor events, perhaps partly because the denominator is reduced, but, of course, ${ }^{3} \mathrm{He}$ and ${ }^{4} \mathrm{He}$ have the same value of FIP. He-poor events are not well understood but they may be the only FIP-dependent variation from event to event (see Chap. 8). If the He-poor SEP events are caused by incomplete FIP processing, which occurs in the chromosphere, then plasma from any CME from the same jet might also be He-poor. The pattern of enhancements for $Z \geq 6$ suggests (minimum $\chi^{2}$ ) a source temperature of $\approx 3 \mathrm{MK}$ where He and $\mathrm{C}$ both have $A / Q=2$, so how else can we decrease $\mathrm{He} / \mathrm{C}$ so greatly?

Are there any impulsive SEP events outside the region 2-4 MK? Reames et al. (2015) could find only a few new events outside 2.5-3.2 MK by relaxing the requirement for high $\mathrm{Fe} / \mathrm{O}$, and none elsewhere. However, Mason et al. (2002a) did find a small ${ }^{3} \mathrm{He}$-rich event with enhanced $\mathrm{N}$ that may have had a temperature of $<1.5 \mathrm{MK}$, but this event was not even visible above $1 \mathrm{MeV} \mathrm{amu}^{-1}$ so it must have 


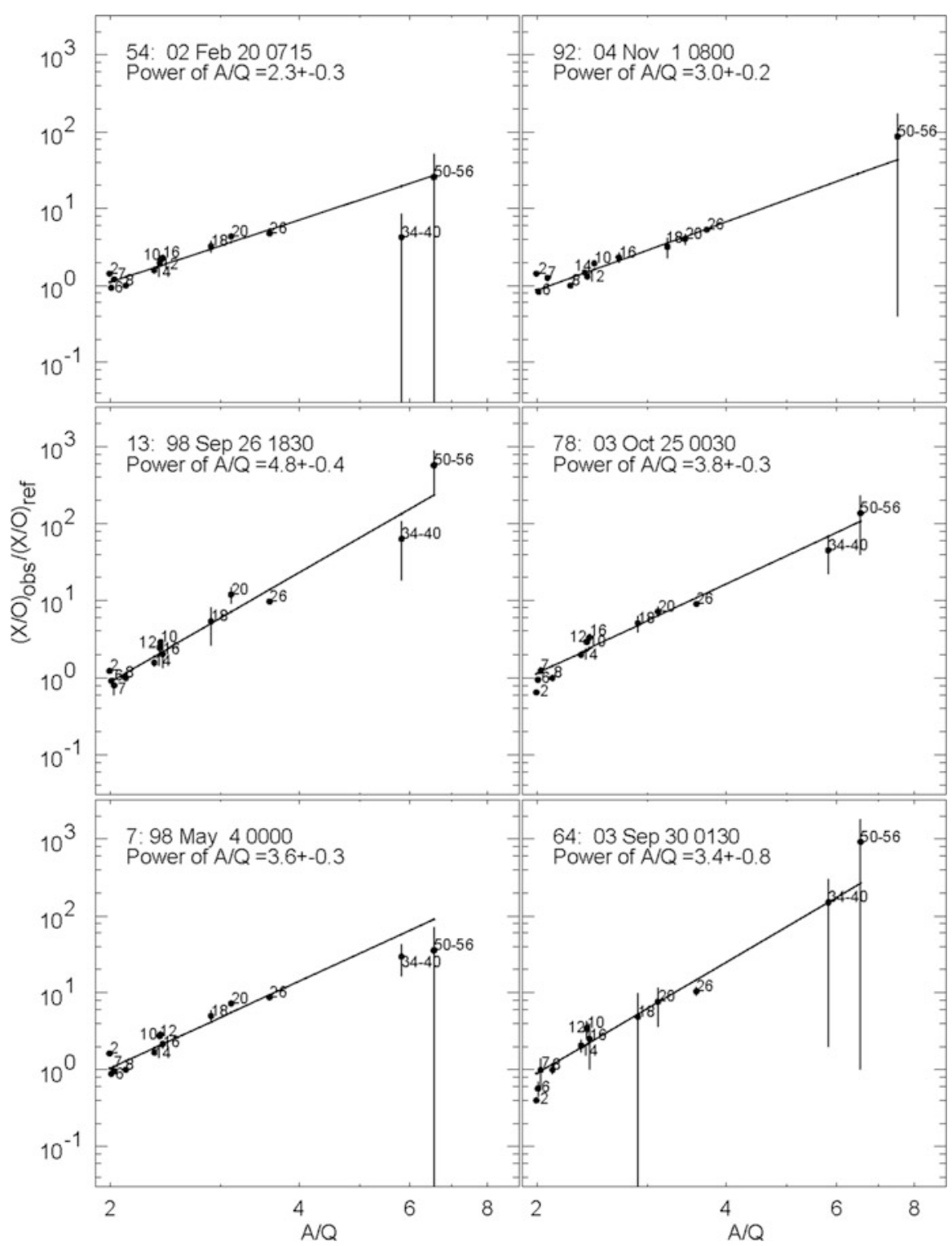

Fig. 4.11 Observed element enhancements relative to corresponding reference values vs. best-fit values of $A / Q$ (at 2.5 or $3.2 \mathrm{MK}$ ) are shown for six individual impulsive SEP events with event numbers (see text), onset times, and power of $A / Q$ shown. Individual points in the panels are labeled with the atomic number $Z$ of the element (Reames 2019, (C) Springer) 
Fig. 4.12 The lower panel shows the time history of several species during two events, 34 and 35, in March 2000. The upper panels show fits to the relative abundances vs. $A / Q$.

Excursions, such as $\mathrm{Ne}$ in the first event and Ar in the second are not shared. However, a large suppression of $\mathrm{He}$ is seen in both events denoted by the arrows (Reames 2019 (C) Springer)
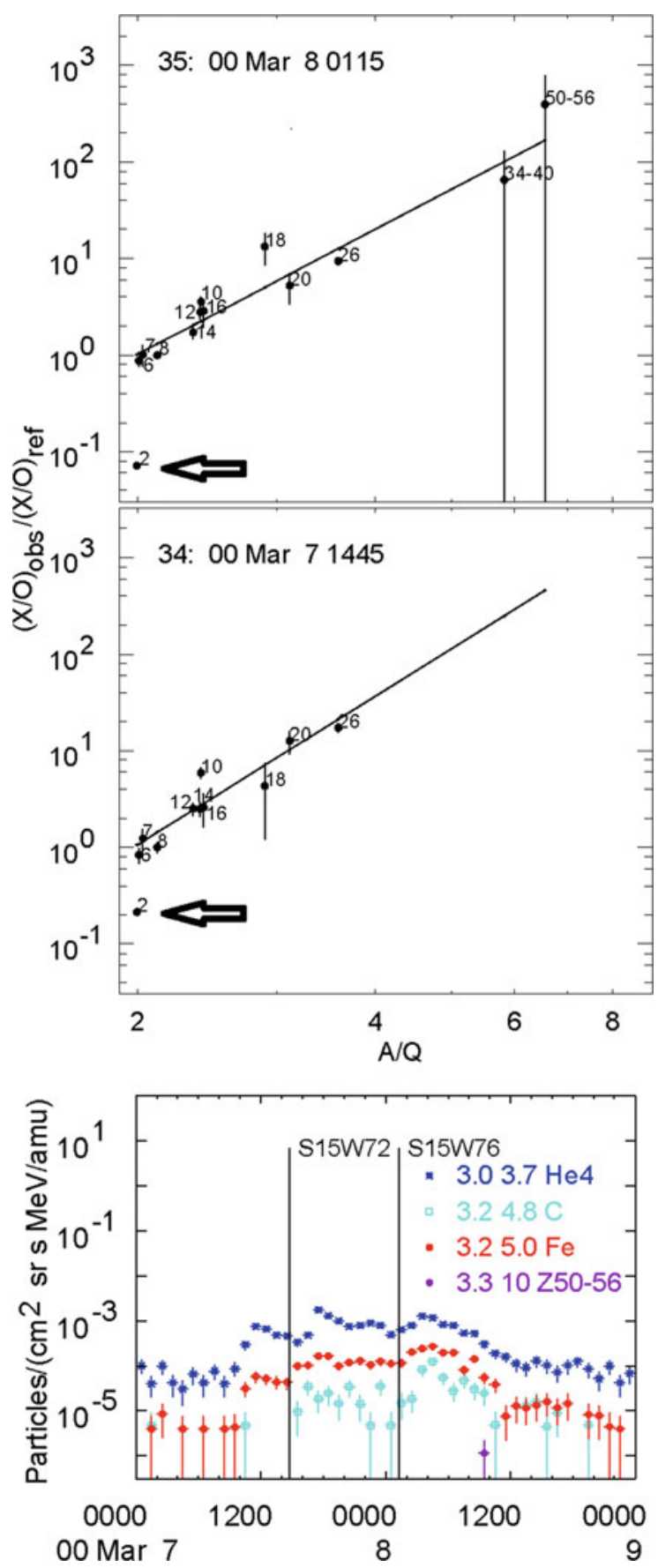
been a very small event with a very steep energy spectrum. Thus, impulsive SEP events outside solar active regions are rare and very small (but see also Sect. 4.8).

\subsection{Associations: CMEs, Flares, and Jets}

While gradual SEP events are associated with fast, wide CMEs, impulsive SEP events are associated with smaller, slower, and especially narrow CMEs. Are these just extremes on a continuum? Probably not, since narrow CMEs from solar jets involve plasma motion along $\boldsymbol{B}$ and may be less likely to produce shocks, while wide ones are from extensive eruptive events that can drive plasma perpendicular to $\boldsymbol{B}$ and produce strong shocks (e.g. Vršnak and Cliver 2008). Nearly $70 \%$ of impulsive Fe-rich SEP events in a recent study (Reames et al. 2014a) have associated CMEs with the properties shown in Fig. 4.13. We often associate impulsive SEP events with flares with the proper timing; flares identify the location of an active region. Flares are carefully tabulated while nearby jets, where the SEPs actually originate, are not. Narrow, slower CMEs are, in fact, associated with jets (Kahler et al. 2001; Bučík et al. 2018a, b). Sources of ${ }^{3}$ He-rich events have been reviewed recently by Bučík (2020).

Any correlation between impulsive-SEP abundances and CMEs is difficult to quantify, but Fig. 4.14 shows enhancements, relative to $\mathrm{He}$, vs. $Z$ for individual events with measured $Z \geq 50$ ions, where the symbols denote the CME width. The events with the greatest enhancements have small, narrow CMEs or no visible CME. Yashiro et al. (2004) previously examined small ${ }^{3} \mathrm{He}$-rich SEP events with no CME and found associated brightness changes and "coronal anomalies" that were probably small CMEs that were too faint to qualify to be cataloged-i.e. they were below threshold. The evidence in Fig. 4.14 suggests that the smaller the CME the greater the enhancements. The smallest events tend to have both suppressed $\mathrm{He} / \mathrm{O}$ and enhanced $(Z \geq 50) / O$.

When abundance enhancements are displayed as a function of the GOES soft X-ray peak intensity as in Fig. 4.15, the smaller B- and C-class events have steeper abundance variations than the brighter $\mathrm{M}$ - and X-class X-ray events. The smaller events are also more likely to have large ${ }^{3} \mathrm{He} /{ }^{4} \mathrm{He}$ ratios (not shown, see Reames et al. 2014b).

As we have said often, flares involve hot closed loops that do not produce SEPs in space. Jets are reconnection events involving open field lines so that plasma and any energetic particles accelerated will be ejected in the diverging field. Thus, it is not at all clear why there should be any correlation of SEP properties with flare heating in the neighboring closed loops of an associated flare, let alone the inverse correlation seen in Fig. 4.15. Apparently it suggests that more-modest reconnection in smaller jets, which may also have minimal flaring, are more likely to produce stronger and steeper abundance enhancements. There is flaring from closing field lines in jets and evaporation of hot plasma from the base of field lines; X-rays must come from hotter regions that differ from the reconnection and SEP source. 
Fig. 4.13 Properties of the impulsive-SEP-associated CMEs and flares are as follows: flare longitude (top), CME width and speed, and the CME-SEP delay (Reames et al. 2014a (C) Springer). The median speed is

$597 \mathrm{~km} \mathrm{~s}^{-1}$ vs. $408 \mathrm{~km} \mathrm{~s}^{-1}$ for all CMEs and $1336 \mathrm{~km} \mathrm{~s}^{-1}$ for gradual SEP events (Yashiro et al. 2004). The average transport delay from CME launch to SEP onset is $2.7 \mathrm{~h}$. From type III onset to SEP onset is $2.3 \mathrm{~h}$, corresponding to a path length of $\sim 1.4 \mathrm{AU}$, which suggests average pitch cosine, $\langle\mu\rangle \approx 0.8$ or a complex path like that in Fig. 3.10
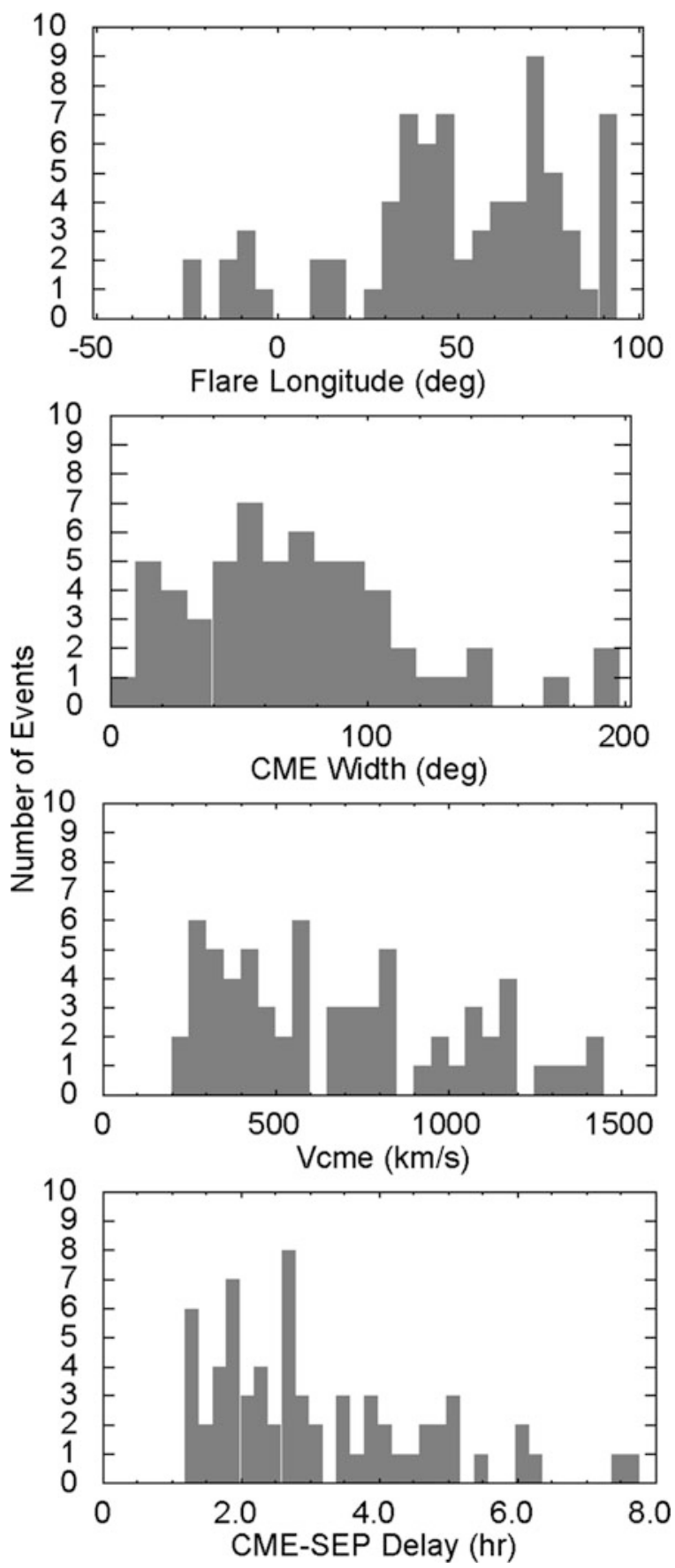


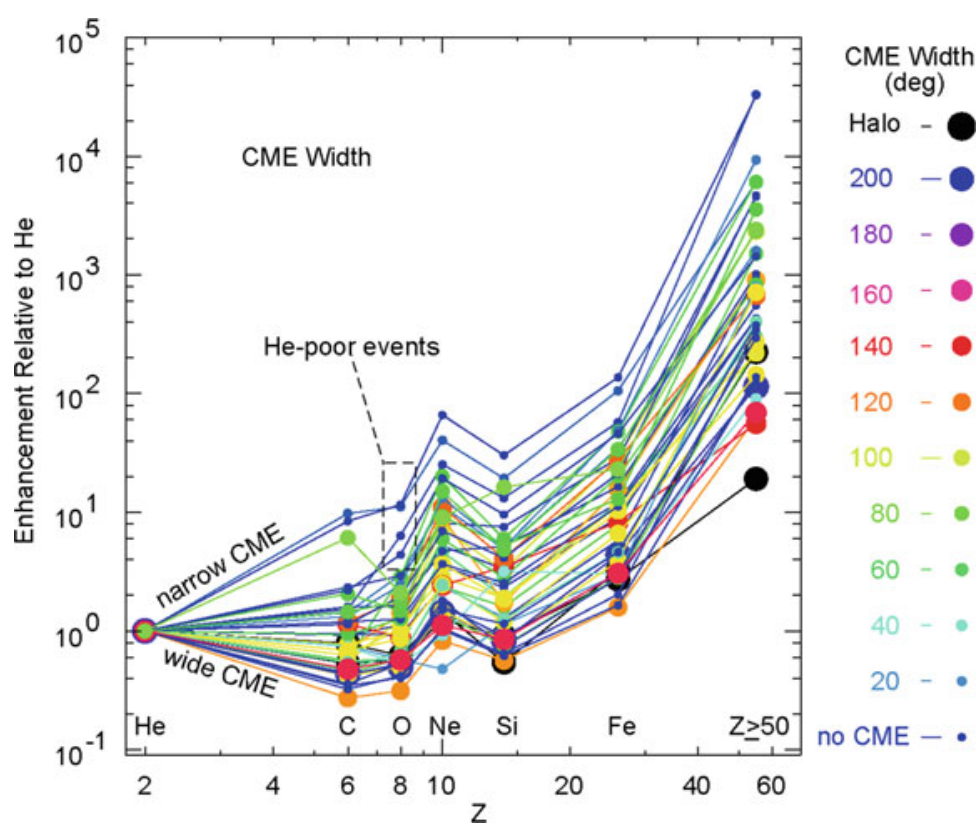

Fig. 4.14 Enhancements relative to He are shown vs. $Z$ for impulsive SEP events. Symbol sizes indicate the associated CME width (Reames et al. 2014a (C) Springer)
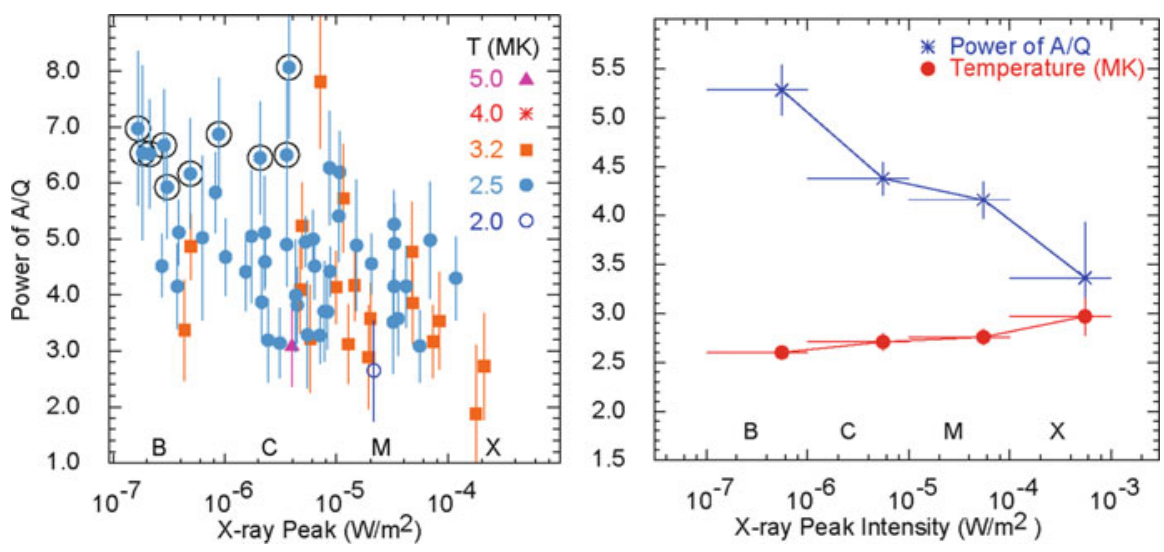

Fig. 4.15 The left panel shows the power of $A / Q$ vs. the GOES soft X-ray peak intensity (and "CMX" class) for individual SEP events with temperature as a symbol and color. Circled events are "He-poor" with low ${ }^{4} \mathrm{He} / \mathrm{O}$ caused only partly by increased $A / Q$ for $\mathrm{O}$. The right panel shows variation of the mean temperature and power of $A / Q$ within each soft X-ray class (after Reames et al. 2014b (C) Springer) 
The cartoon in Fig. 4.16 illustrates the basic mechanism behind a jet produced when new magnetic flux (blue) emerges, pressing into oppositely directed open magnetic field (black). The time evolution of an isolated jet is shown in Fig. 4.17.

The drawing in Fig. 4.16 shows a reconnection region formed when emerging magnetic flux is oppositely directed from the overlying flux. The magnetic reconnection region is non-uniform and forms islands of reconnection. Oppositely-

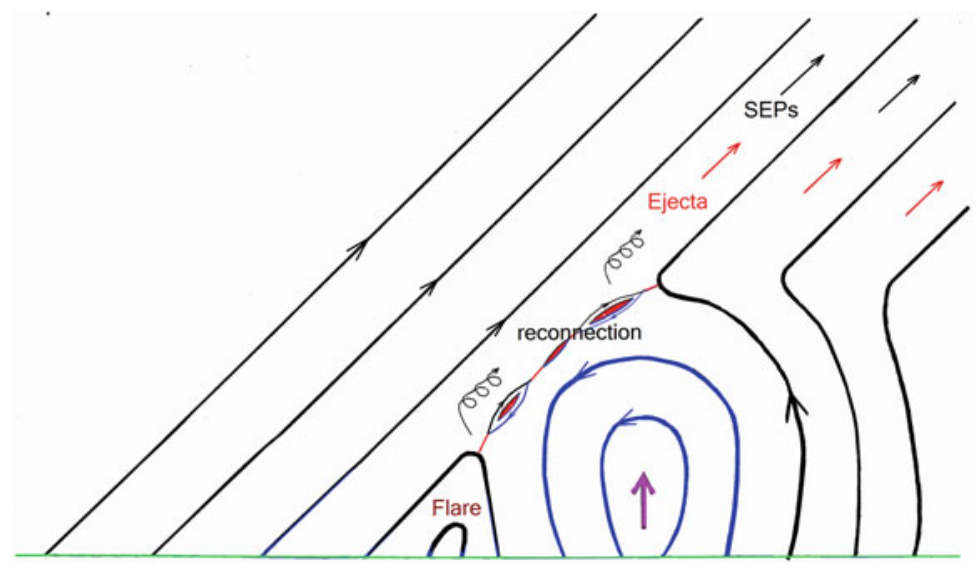

Fig. 4.16 A jet is produced when newly emerging magnetic flux (blue) reconnects with oppositely directed field (black) in the red region. The reconnection region is not a uniform surface but forms islands of reconnection. Energetic particles and plasma can escape toward the upper right; an enclosed flaring region forms at the lower left (see Reames 2002). Real jets can be much more complex, involving twisted fields, etc. (see review by Raouafi et al. 2016)

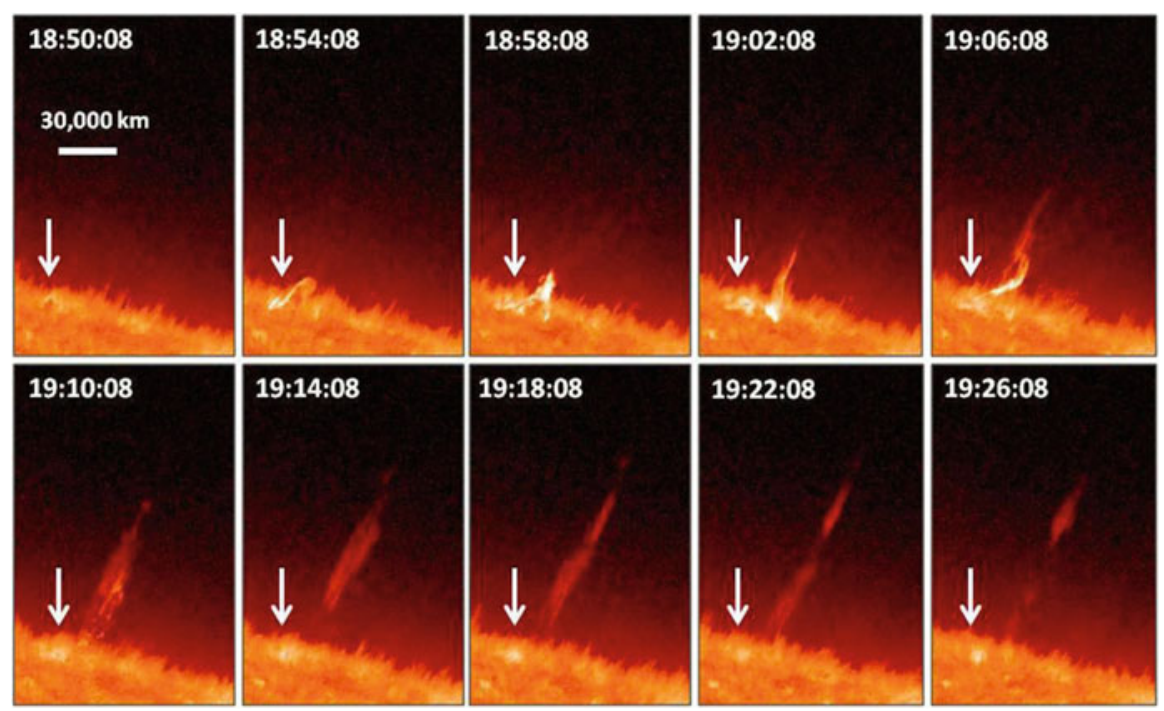

Fig. 4.17 Eruption of an isolated jet on 11 August 2010 observed as a function of time on SDO/AIA of He II at 304 Å by Moore, Sterling, and Falconer (2015 (C) AAS) 
directed fields do not perfectly cancel, and a small out-of-plane "guide field" may stabilize the reconnection. Particle-in-cell simulations show Fermi acceleration of ions reflected back and forth from the ends of the collapsing islands of reconnection (Drake et al. 2009). If the distribution of the widths $w$ of islands of reconnection is $P$ (w) $\sim w^{-\alpha}$, then the rate of production of energetic ions with mass $A$ and charge $Q$ is.

$$
d N / d t \sim w_{t h}^{3-\alpha} \sim(A / Q)^{\alpha-3}
$$

where $w_{t h}$ is the threshold for ion heating which typically occurs when $A / Q>5$ (Drake et al. 2009). However, we see enhancements all the way from $A / Q=1$.

The theory of ion (other than ${ }^{3} \mathrm{He}$, covered in Sect. 2.5.2) and electron acceleration in flares is extensive (see Ramaty 1979; Steinacker et al. 1993; Miller et al. 1997; Miller 1998). Stochastic acceleration of ions by an arbitrary wave spectrum is common and the balance between acceleration and Coulomb losses (e.g. Mason and Klecker 2018) has been considered for nearly 40 years (Ramaty 1979). However, the observation that ${ }^{4} \mathrm{He}$ and $\mathrm{C}$ are both unenhanced and have $A / Q=2$ at $T \approx 3 \mathrm{MK}$ seems to argue against a dependence upon $A / Q^{2}$ that would be appropriate for traversal of material. We tend to favor the particle-in-cell results (Drake et al. 2009) because they seem more directly related to reconnection and jets.

X-ray properties of jets were described by Shimojo and Shibata (2000) and they were associated with impulsive SEP events by Kahler et al. (2001). X-ray jets were also previously associated with type III radio bursts which provide the streaming electrons that may generate the EMIC waves needed for ${ }^{3} \mathrm{He}$ enhancements (Temerin and Roth 1992; Roth and Temerin 1997). The narrow CMEs associated with two impulsive SEP events are shown in Fig. 4.18.

It has also been possible to trace the magnetic field lines from Earth to the Sun to locate the sources of events (Wang et al. 2006; Nitta et al. 2006). Much more sophisticated models of jets have also evolved that show reconnection associated with untwisting of axial field lines and generation of Alfvén waves (Moore et al.

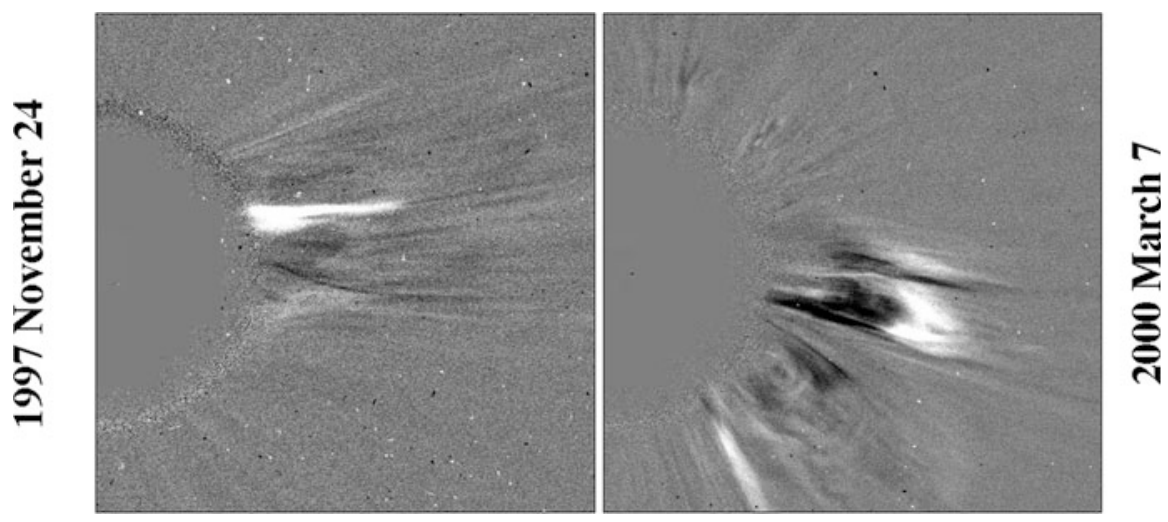

Fig. 4.18 Difference images show change in intensity in the LASCO C2 coronagraph for narrow CMEs associated with impulsive SEP events (Kahler et al. 2001 (C) AAS) 
2013; Lee et al. 2015). However, such models do not yet include any related particle acceleration (see review of solar jets by Raouafi et al. 2016) although observations now clearly associate ${ }^{3} \mathrm{He}$-rich SEP events directly with helical jets (Bučík et al. 2018a, b; Bučík 2020). These SEP events are often associated with active-region coronal holes (Innes et al. 2016), i.e. open field lines extending out of an active region. Active-region jets tend to have temperatures of $\sim 3 \mathrm{MK}$ (see Fig. 14 of Raouafi et al. 2016) like those we deduce from impulsive SEP events (Fig. 4.10).

Recently, Paraschiv and Donea (2019; Paraschiv 2018) have associated recurrent solar jets with coronal "geysers", magnetic structures that are observed to generate as many as a dozen individual jets over a period of a day or so. These jets are found to produce the electron beams that generate type-III radio bursts (Sect. 2.2) which we associate with impulsive SEP events (e.g. Sect. 2.5.2). Geysers may be involved in the long periods of ${ }^{3} \mathrm{He}$-rich, Fe-rich suprathermal ions that are observed (Fig. 2.8). It is well known that multiple impulsive SEP events commonly occur together on this time scale (see e.g. Fig. 4.2). Magnetic flux emergence is still regarded as an important trigger mechanism for solar jets (Paraschiv et al. 2020).

The studies of ion acceleration in islands of magnetic reconnection come from particle-in-cell simulations discussed above (Drake et al. 2009; Knizhnik et al. 2011; Drake and Swisdak 2012). This acceleration produces a strong power-law dependence on $A / Q$ and provides the most promising explanation of the element abundance enhancements in impulsive SEP events appropriate to solar jets. However, ${ }^{3} \mathrm{He}$ enhancements seem to require a separate explanation involving resonant waves (e.g. Temerin and Roth 1992).

\subsection{Can We Have It Both Ways?}

It seems suspicious to derive ${ }^{3} \mathrm{He}$ enhancements from one mechanism and heavyelement enhancements from another-in the same SEP event. Do both really contribute?

Recently, Mason et al. (2016) found $16{ }^{3} \mathrm{He}$-rich events in 16 years with extremely high $\mathrm{S} / \mathrm{O}$ abundances in the $0.4-1.0 \mathrm{MeV}_{\mathrm{amu}}{ }^{-1}$ interval. Most of these events are too small and their spectra too steep to be measurable above 1.0 $\mathrm{MeV} \mathrm{amu}^{-1}$, the few we can measure show no significant anomalies. Properties of the most extreme event of 16 May 2014 are shown in Fig. 4.19.

This event has ${ }^{3} \mathrm{He} /{ }^{4} \mathrm{He}=14.88 \pm 1.36$ and $\mathrm{S} / \mathrm{O}=1.14 \pm 0.12$, although the spectra show that these abundances are not constant but vary with energy. The shapes of the spectra of $\mathrm{Si}, \mathrm{S}$, and $\mathrm{Fe}$ are similar to that of ${ }^{3} \mathrm{He}$, strongly suggesting that these elements are all accelerated or modified by the same mechanism.

Mason et al. (2016) consider a wide range of plasma temperatures above 0.4 MK. However, most models of resonant wave-particle acceleration (e.g. Fisk 1978; Roth and Temerin 1997) suggest that heavy elements might resonate with the same waves as ${ }^{3} \mathrm{He}$, but through the second harmonic of their gyrofrequency. Since $A /$ $Q=1.5$ for ${ }^{3} \mathrm{He}$, naively we might expect $\mathrm{Si}$ and $\mathrm{S}$ to be accelerated when they have $A / Q \approx 3.0$, if the resonance is broad enough for us to use an average value of $A / Q$. 

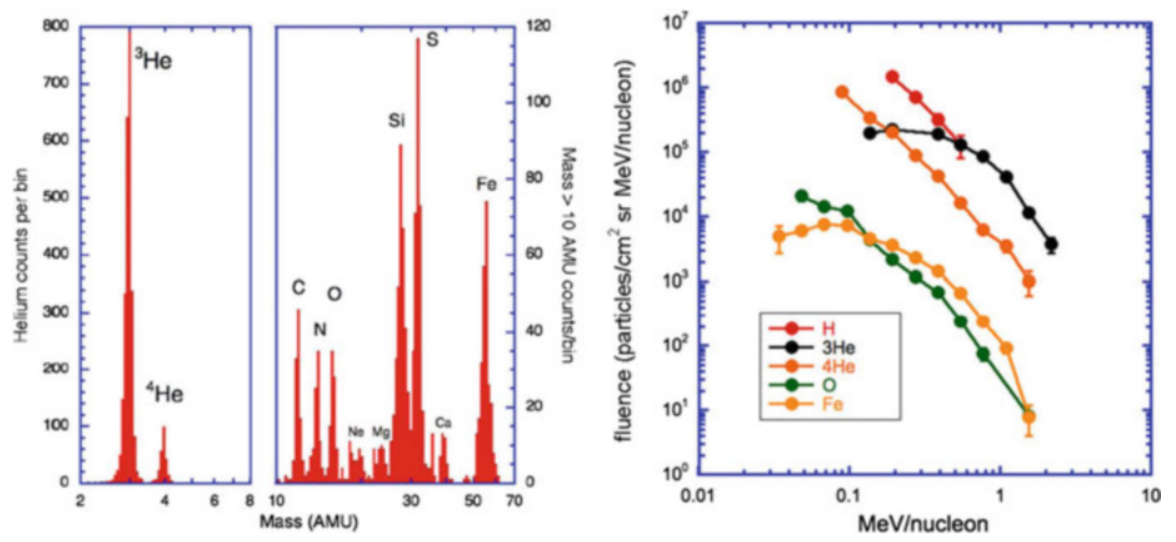

Fig. 4.19 Mass histograms of the 16 May $2014{ }^{3} \mathrm{He}$-rich event are shown in the left two panels and some corresponding energy spectra are shown in the right panel (Mason et al. 2016 (C) AAS)

For S, this average value occurs at 2.0 MK and for Si near 1.5 MK, both reasonable temperatures that might exist near the fringes of active regions. Solar jets near active regions have temperatures around $3 \mathrm{MK}$ while those from coronal holes are nearer 1.5 MK (see Fig. 14 of Raouafi et al. 2016). Jets from coronal holes might also be based upon different FIP-dependent coronal abundances like those in the solar wind where both $\mathrm{C}$ and $\mathrm{S}$ behave like a low-FIP elements (see Sects. 8.4 and 8.5; Reames 2020). This increased factor of $\sim 4$ in S/O would only partly explain the observations, but the elevated C/O may be a clue. However, the greatly enhanced $\mathrm{S}$ does not seem to be supported at 2.0 MK by Roth and Temerin (1997) either. Present theory of ${ }^{3} \mathrm{He}$ involves resonance of ions with electron-beam-generated EMIC waves; direct wave generation in the reconnection region is also a possibility that has not yet been explored.

The enhancements of other elements with less-rounded spectra might result from the power-law in $A / Q$ more-commonly produced at higher energies by magnetic reconnection (e.g. Drake et al. 2009). It seems possible that the two mechanisms may compete to dominate different species at different energies in different impulsive SEP events, but a clear picture of the physics is still elusive and the degree of enhancement of all species may not be easily accommodated.

The understanding of the relative roles of the reconnection and the resonant wave mechanisms is the largest outstanding problem in the physics of flares, jets, and impulsive SEP events. Nature has tempted us with two huge 1000-fold enhancements, in ${ }^{3} \mathrm{He}$ and in heavy elements. Neither one is subtle. Yet they seem to be unrelated and we are unable to incorporate their explanations into a single physical model that can tell us which will dominate and when. 


\subsection{Nuclear Reactions: Gamma-Ray Lines and Neutrons}

It may seem incongruous to discuss $\gamma$-ray-line events in a chapter on impulsive SEP events since $\gamma$-ray lines have not been observed in small events or jets. Line emission is observed in large flares from the de-excitation of nuclei produced in nuclear reactions that occur when ions, accelerated on closed coronal loops, are scattered into the loss cone and plunge into the higher-density corona (e.g. Ramaty and Murphy 1987; Kozlovsky et al. 2002). Hard (>20 keV) X-rays, common in flares, are produced by non-relativistic electrons but X-rays tell us nothing about accelerated ions; only $\gamma$-ray lines can help here. Protons, undergoing nuclear reactions with $\mathrm{C}, \mathrm{O}$, and $\mathrm{Fe}$, for example, produce narrow $\gamma$-ray spectral lines in the region of $\sim 0.5-7 \mathrm{MeV}$, whose relative intensities can be used to measure abundances of elements in the corona. Energetic heavy ions in the "beam" interacting with protons in the corona produce Doppler-broadened spectral lines that measure abundances in the accelerated beam, i.e. the flare equivalent of our SEPs. Figure 4.20, from Murphy et al. (1991) compares observed and calculated $\gamma$ ray spectra in the large event of 27 April 1981.

These lines suggest that the accelerated ions in large flares are both ${ }^{3} \mathrm{He}$-rich (Mandzhavidze et al. 1999) and Fe-rich (Murphy et al. 1991). The $\gamma$-ray lines excited by nuclear reactions of a ${ }^{3} \mathrm{He}$ beam differ from those produced by a ${ }^{4} \mathrm{He}$ beam, allowing measurement of both isotopes. The presence of enhanced ${ }^{3} \mathrm{He} /{ }^{4} \mathrm{He}$ in the accelerated "beam" in these events has been further confirmed recently by Murphy et al. (2016). Thus it seems that flares and jets might accelerate ions in the same way and $\gamma$-ray-line spectroscopy could contribute to our understanding of that process. Unfortunately, the masks used to improve position resolution in modern
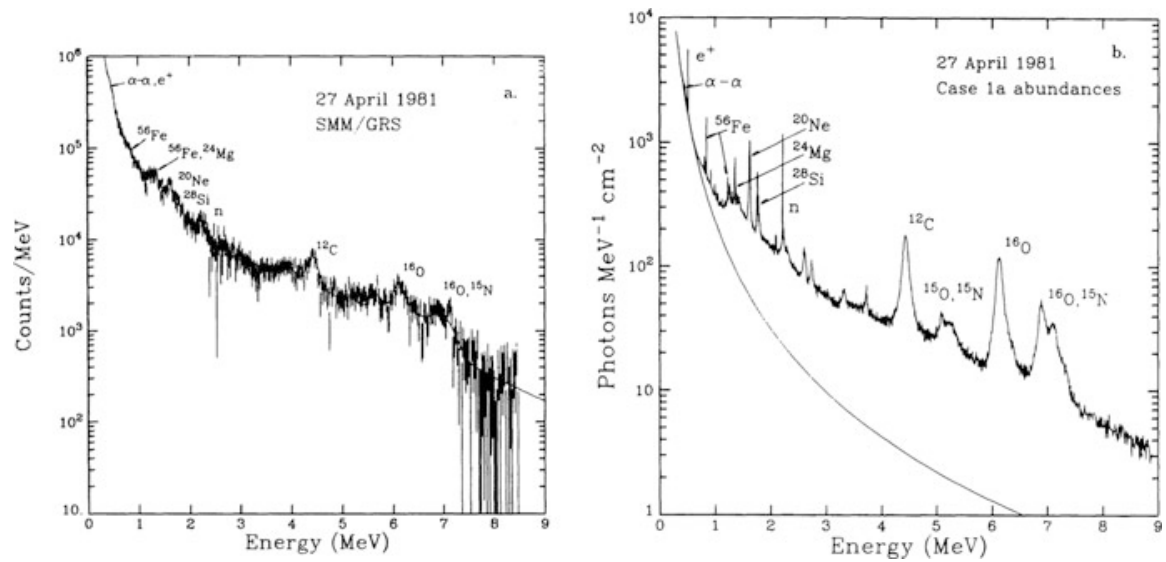

Fig. 4.20 An observed $\gamma$-ray spectrum (a) is compared with a calculated spectrum (b). Relative strengths of the lines are determined by element abundances in the corona and in the accelerated "beam" (Murphy et al. 1991 (C) AAS) 
spectroscopy missions, which operate rather like a pin-hole camera, block too many $\gamma$-rays to permit $\gamma$-ray-line spectroscopy on modern spacecraft.

Nuclear reactions in the corona also produce ${ }^{2} \mathrm{H},{ }^{3} \mathrm{H}$, positrons, $\pi$-mesons, and isotopes of $\mathrm{Li}, \mathrm{Be}$, and $\mathrm{B}$ as inferred from the $\gamma$-ray-line spectroscopy. However, as we re-emphasize, none of these secondary products (except $\gamma$-rays and neutrons) has been observed into space. Apparently the secondary ions are magnetically trapped on the loops where they are created, suggesting that the primary ions that produced them were similarly trapped.

Evidence of a spatially extended $\gamma$-ray source and long-duration $\gamma$-ray events are discussed in Sect. 5.7. We will see that the $>100 \mathrm{MeV} \gamma$-rays are produced by $>300 \mathrm{MeV}$ protons accelerated by CME-driven shock waves (Plotnikov et al. 2017; Share et al. 2018).

Neutrons are also produced in nuclear reactions in solar flares and $50-300 \mathrm{MeV}$ neutrons have been observed directly in space (Chupp et al. 1982; Chupp 1984). Neutrons decay into a proton, electron, and neutrino with a 10-min half life and neutron-decay protons of 5-200 MeV have also been measured, allowing a neutron spectrum to be calculated (e.g. Evenson et al. 1983, 1990). Neutron-decay protons are best measured for eastern solar events where they can be measured on field lines that are much less accessible to protons directly from the shock source, which slowly increase later.

\subsection{Open Questions}

This section suggests open questions that might be addressed by future research.

1. If most $A / Q$ enhancements come from magnetic reconnection and ${ }^{3} \mathrm{He}$ enhancements come from wave-particle interactions, where and when do these two acceleration mechanisms fit into models of solar jets? What parameters control their relative contributions?

2. Why do the impulsive SEP events with the lowest intensities, smallest associated flares and narrowest CMEs have the greatest enhancements of ${ }^{3} \mathrm{He} /{ }^{4} \mathrm{He}$ and the heavy-element abundance enhancements with the steepest power of $A / Q$ ? To what extent can all the ${ }^{3} \mathrm{He}$ event observations be explained by source depletion of the enhanced species? Surely not Fe-enhancements?

3. Measurements by a spacecraft near the Sun, like PSP, could improve SEP onset timing by removing the blurring effect of scattering during transport. What is the duration and time profile of impulsive events at $10 \mathrm{~s}$ to $1 \mathrm{~min}$ resolution and how does it compare with X-ray, $\gamma$-ray, and type-III-burst timing? Note that intensities may vary as $\sim r^{-3}$, providing greatly improved statistics nearer the Sun for smaller events, but anisotropies may be high. Do the electron and ion sources differ? What is the relative timing of ${ }^{3} \mathrm{He}$ and ${ }^{4} \mathrm{He}$ ? 
4. For a spacecraft near the Sun, the $\sim r^{-3}$ intensity increase would allow observation of many more small impulsive SEP events from smaller jets. What is the size distribution? Are there microjets and nanojets supplying many impulsive suprathermal ions during times that are otherwise quiet?

5. Discrete ionization states affect the assignment of source-plasma temperatures. ${ }^{12} \mathrm{C}^{+5}$ is enhanced but ${ }^{12} \mathrm{C}^{+6}$ is not; treating $Q$ as 5.5 is approximate. $A /<Q>$ is not the same as $\langle A / Q\rangle$. Then there is ${ }^{13} \mathrm{C}$ which is always enhanced. Can we improve the estimates of $T$ ?

6. $\mathrm{Can}^{22} \mathrm{Ne}$ enhancement explain the occasional extra enhancements of $\mathrm{Ne}$ ?

7. What causes the large enhancements of $\mathrm{S}$ in some small impulsive events? Are the events connected to active regions?

8. What causes the occasional large suppression in the He-poor events?

\section{References}

Arnaud, M., Raymond, J.: Iron ionization and recombination rates and ionization equilibrium. Astrophys. J. 398, 394 (1992). https://doi.org/10.1086/171864

Arnaud, M., Rothenflug, R.: An updated evaluation of recombination and ionization rates. Astron. Astrophys. Suppl. 60, 425 (1985)

Bučík, R.: ${ }^{3}$ He-rich solar energetic particles: solar sources. Space Sci. Rev. 216, 24 (2020). https:// doi.org/10.1007/s11214-020-00650-5

Bučík, R., Innes, D.E., Mason, G.M., Wiedenbeck, M.E., Gómez-Herrero, R., Nitta, N.: ${ }^{3}$ He-rich solar energetic particles in helical jets on the sun. Astrophys. J. 852, 76 (2018a). https://doi.org/ 10.3847/1538-4357/aa9d8f

Bučík, R., Wiedenbeck, M.E., Mason, G.M., Gómez-Herrero, R., Nitta, N.V., Wang, L.: ${ }^{3}$ He-rich solar energetic particles from sunspot jets. Astrophys. J. Lett. 869, L21 (2018b). https://doi.org/ 10.3847/2041-8213/aaf37f

Chupp, E.L.: High-energy neutral radiations from the sun. Ann. Rev. Astron. Astrophys. 22, 359 (1984). https://doi.org/10.1146/annurev.aa.22.090184.002043

Chupp, E.L., Forrest, D.J., Ryan, J.M., Heslin, J., Reppin, C., Pinkau, K., Kanbach, G., Rieger, E., Share, G.H.: A direct observation of solar neutrons following the 0118 UT flare on 1980 June 21, Astrophys. J. Lett. 263, L95 (1982). https://doi.org/10.1086/183931

Cook, W.R., Stone, E.C., Vogt, R.E.: Elemental composition of solar energetic particles. Astrophys. J. 279, 827 (1984). https://doi.org/10.1086/161953

Drake, J.F., Swisdak, M.: Ion heating and acceleration during magnetic reconnection relevant to the corona. Space Sci. Rev. 172, 227 (2012). https://doi.org/10.1007/s11214-012-9903-3

Drake, J.F., Cassak, P.A., Shay, M.A., Swisdak, M., Quataert, E.: A magnetic reconnection mechanism for ion acceleration and abundance enhancements in impulsive flares. Astrophys. J. Lett. 700, L16 (2009). https://doi.org/10.1088/0004-637X/700/1/L16

Evenson, P., Meyer, P., Pyle, K.R.: Protons from the decay of solar flare neutrons. Astrophys. J. 274, 875 (1983). https://doi.org/10.1086/161500

Evenson, P., Kroeger, R., Meyer, P., Reames, D.: Solar neutron decay proton observations in cycle 21. Astrophys. J. Suppl. 73, 273 (1990). https://doi.org/10.1086/191462

Fisk, L.A.: ${ }^{3}$ He-rich flares - a possible explanation. Astrophys. J. 224, 1048 (1978). https://doi.org/ $10.1086 / 156456$

Innes, D.E., Bučík, R., Gao, L.-J., Nitta, N.: Observations of solar X-ray and EUV jets and their related phenomena. Astronomische Nachrichten. 337, 1024 (2016). https://doi.org/10.1002/ asna. 201612428 
Kahler, S.W., Reames, D.V., Sheeley Jr., N.R.: Coronal mass ejections associated with impulsive solar energetic particle events. Astrophys. J. 562, 558 (2001). https://doi.org/10.1086/323847

Knizhnik, K., Swisdak, M., Drake, J.F.: The acceleration of ions in solar flares during magnetic reconnection. Astrophys. J. Lett. 743, L35 (2011). https://doi.org/10.1088/2041-8205/743/2/ L35

Kozlovsky, B., Murphy, R.J., Ramaty, R.: Nuclear deexcitation gamma-ray lines from accelerated particle interactions. Astrophys. J. Suppl. 141, 523 (2002). https://doi.org/10.1086/340545

Lee, E.J., Archontis, V., Hood, A.W.: Helical blowout jets in the sun: untwisting and propagation of waves. Astrophys. J. Lett. 798, L10 (2015). https://doi.org/10.1088/2041-8205/798/1/L10

Liu, S., Petrosian, V., Mason, G.M.: stochastic acceleration of ${ }^{3} \mathrm{He}$ and ${ }^{4} \mathrm{He}$ in solar flares by parallel-propagating plasma waves: general results. Astrophys. J. 636, 462 (2006). https://doi. org/10.1086/497883

Mandzhavidze, N., Ramaty, R., Kozlovsky, B.: Determination of the abundances of subcoronal ${ }^{4} \mathrm{He}$ and of solar flare-accelerated ${ }^{3} \mathrm{He}$ and ${ }^{4} \mathrm{He}$ from gamma-ray spectroscopy. Astrophys. J. 518, 918 (1999). https://doi.org/10.1086/307321

Mason, G.M.: ${ }^{3}$ He-rich solar energetic particle events. Space Sci. Rev. 130, 231 (2007). https://doi. org/10.1007/s11214-007-9156-8

Mason, G.M., Klecker, B.: A possible mechanism for enriching heavy ions in ${ }^{3} \mathrm{He}-$ rich solar energetic particle events. Astrophys. J. 862, 7 (2018). https://doi.org/10.3847/1538-4357/ aac94c

Mason, G.M., Reames, D.V., Klecker, B., Hovestadt, D., von Rosenvinge, T.T.: The heavy-ion compositional signature in He-3-rich solar particle events. Astrophys. J. 303, 849 (1986). https:// doi.org/10.1086/164133

Mason, G.M., Mazur, J.E., Dwyer, J.R.: A new heavy ion abundance enrichment pattern in ${ }^{3}$ He-rich solar particle events. Astrophys. J. Lett. 565, L51 (2002a). https://doi.org/10.1086/339135

Mason, G.M., Wiedenbeck, M.E., Miller, J.A., Mazur, J.E., Christian, E.R., Cohen, C.M.S., Cummings, A.C., Dwyer, J.R., Gold, R.E., Krimigis, S.M., Leske, R.A., Mewaldt, R.A., Slocum, P.L., Stone, E.C., von Rosenvinge, T.T.: Spectral properties of He and heavy ions in ${ }^{3}$ He-rich solar flares. Astrophys. J. 574, 1039 (2002b). https://doi.org/10.1086/341112

Mason, G.M., Mazur, J.E., Dwyer, J.R., Jokipii, J.R., Gold, R.E., Krimigis, S.M.: Abundances of heavy and ultraheavy ions in ${ }^{3}$ He-rich solar flares. Astrophys. J. 606, 555 (2004). https://doi.org/ $10.1086 / 382864$

Mason, G.M., Nitta, N.V., Wiedenbeck, M.E., Innes, D.E.: Evidence for a common acceleration mechanism for enrichments of ${ }^{3} \mathrm{He}$ and heavy ions in impulsive SEP events. Astrophys. J. 823, 138 (2016). https://doi.org/10.3847/0004-637X/823/2/138

Miller, J.A.: Particle acceleration in impulsive solar flares. Space Sci. Rev. 86, 79 (1998). https:// doi.org/10.1023/A:1005066209536

Miller, J.A., Cargill, P.J., Emslie, A.G., Holman, G.D., Dennis, B.R., LaRosa, T.N., Winglee, R.M., Benka, S.G., Tsuneta, S.: Critical issues for understanding particle acceleration in impulsive solar flares. J. Geophys. Res. 102, 14631 (1997). https://doi.org/10.1029/97JA00976

Moore, R.L., Sterling, A.C., Falconer, D.A., Robe, D.: The cool component and the dichotomy, lateral expansion, and axial rotation of solar X-ray jets. Astrophys. J. 769, 134 (2013). https:// doi.org/10.1088/0004-637X/769/2/134

Moore, R.L., Sterling, A.C., Falconer, D.A.: Magnetic untwisting in solar jets that go into the outer corona in polar coronal holes. Astrophys. J. 806, 11 (2015). https://doi.org/10.1088/0004-637X/ 769/2/134

Murphy, R.J., Ramaty, R., Kozlovsky, B., Reames, D.V.: Solar abundances from gamma-ray spectroscopy: comparisons with energetic particle, photospheric, and coronal abundances. Astrophys. J. 371, 793 (1991). https://doi.org/10.1086/169944

Murphy, R.J., Kozlovsky, B., Share, G.H.: Evidence for enhanced ${ }^{3}$ He in flare-accelerated particles based on new calculations of the gamma-ray line spectrum. Astrophys. J. 833, 196 (2016). https://doi.org/10.3847/1538-4357/833/2/196 
Nitta, N.V., Reames, D.V., DeRosa, M.L., Yashiro, S., Gopalswamy, N.: Solar sources of impulsive solar energetic particle events and their magnetic field connection to the Earth. Astrophys. J. 650, 438 (2006). https://doi.org/10.1086/507442

Paraschiv, A.R.: On long timescale recurrent active region coronal jets: the coronal geyser structure, Ph.D. thesis, Monash University (2018). doi: https://doi.org/10.26180/5bc9d76627396

Paraschiv, A.R., Donea, A.: On solar recurrent coronal jets: coronal geysers as sources of electron beams and interplanetary type-III radio bursts. Astrophys. J. 873, 110 (2019). https://doi.org/10. 3847/1538-4357/ab04a6

Paraschiv, A.R., Donea, A., Leka, K.D.: The trigger mechanism of recurrent solar active region jets revealed by the magnetic properties of a coronal geyser site. Astrophys. J. 891, 149 (2020). https://doi.org/10.3847/1538-4357/ab7246

Plotnikov, I., Rouillard, A., Share, G.: The magnetic connectivity of coronal shocks to the visible disk during long-duration gamma-ray flares. Astron. Astrophys. 608, 43 (2017). https://doi.org/ 10.1051/0004-6361/201730804. (arXiv:1703.07563)

Post, D.E., Jensen, R.V., Tarter, C.B., Grasberger, W.H., Lokke, W.A.: Steady-state radiative cooling rates for low-density, high temperature plasmas. At. Data Nucl. Data Tables. 20, 397 (1977). https://doi.org/10.1016/0092-640X(77)90026-2

Ramaty, R.: Energetic particles in solar flares. AIP Conf. Proc. 56, 135 (1979). https://doi.org/10. $1063 / 1.32074$

Ramaty, R., Murphy, R.J.: Nuclear processes and accelerated particles in solar flares. Space Sci. Rev. 45, 213 (1987). https://doi.org/10.1007/BF00171995

Raouafi, N.E., Patsourakos, S., Pariat, E., Young, P.R., Sterling, A.C., Savcheva, A., Shimojo, M., Moreno-Insertis, F., DeVore, C.R., Archontis, V., et al.: Solar coronal jets: observations, theory, and modeling. Space Sci. Rev. 201, 1 (2016). https://doi.org/10.1007/s11214-016-0260-5. (arXiv:1607.02108)

Reames, D.V.: Bimodal abundances in the energetic particles of solar and interplanetary origin. Astrophys. J. Lett. 330, L71 (1988). https://doi.org/10.1086/185207

Reames, D.V.: Coronal Abundances determined from energetic particles. Adv. Space Res. 15(7), $41(1995)$

Reames, D.V.: Particle acceleration at the Sun and in the Heliosphere. Space Sci. Rev. 90, 413 (1999). https://doi.org/10.1023/A:1005105831781

Reames, D.V.: Abundances of trans-iron elements in solar energetic particle events. Astrophys. J. Lett. 540, L111 (2000). https://doi.org/10.1086/312886

Reames, D.V.: Magnetic topology of impulsive and gradual solar energetic particle events. Astrophys. J. Lett. 571, L63 (2002). https://doi.org/10.1086/341149

Reames, D.V.: Helium suppression in impulsive solar energetic-particle events. Sol. Phys. 294, 32 (2019). https://doi.org/10.1007/s11207-019-1422-x. (arXiv: 1812.01635)

Reames, D.V.: Four distinct pathways to the element abundances in solar energetic particles. Space Sci. Rev. 216, 20 (2020). https://doi.org/10.1007/s11214-020-0643-5

Reames, D.V., Ng, C.K.: Heavy-element abundances in solar energetic particle events. Astrophys. J. 610, 510 (2004). https://doi.org/10.1086/421518

Reames, D.V., Meyer, J.P., von Rosenvinge, T.T.: Energetic-particle abundances in impulsive solar flare events. Astrophys. J. Suppl. 90, 649 (1994). https://doi.org/10.1086/191887

Reames, D.V., Cliver, E.W., Kahler, S.W.: Abundance enhancements in impulsive solar energeticparticle events with associated coronal mass ejections. Sol. Phys. 289, 3817 (2014a). https://doi. org/10.1007/s11207-014-0547-1. (arXiv: 1404.3322)

Reames, D.V., Cliver, E.W., Kahler, S.W.: Variations in abundance enhancements in impulsive solar energetic-particle events and related CMEs and flares. Sol. Phys. 289, 4675 (2014b). https://doi.org/10.1007/s11207-014-0589-4

Reames, D.V., Cliver, E.W., Kahler, S.W.: Temperature of the source plasma for impulsive solar energetic particles. Sol. Phys. 290, 1761 (2015). https://doi.org/10.1007/s11207-015-0711-2

Roth, I., Temerin, M.: Enrichment of ${ }^{3} \mathrm{He}$ and heavy ions in impulsive solar flares. Astrophys. J. 477, 940 (1997). https://doi.org/10.1086/303731 
Serlemitsos, A.T., Balasubrahmanyan, V.K.: Solar particle events with anomalously large relative abundance of ${ }^{3}$ He. Astrophys. J. 198, 195 (1975). https://doi.org/10.1086/153592

Share, G.H., Murphy, R.J., White, S.M., Tolbert, A.K., Dennis, B.R., Schwarz, R.A., Smart, D.F., Shea, M.A.: Characteristics of late-phase $>100 \mathrm{MeV} \gamma$-ray emission in solar eruptive events. Astrophys. J. 869, 182 (2018). https://doi.org/10.3847/1538-4357/aaebf7

Shimojo, M., Shibata, K.: Physical parameters of solar X-ray jets. Astrophys. J. 542, 1100 (2000). https://doi.org/10.1086/317024

Steinacker, J., Jaeckel, U., Schlickeiser, R.: Ion acceleration in impulsive solar flares. Astrophys. J. 415, 342 (1993). https://doi.org/10.1086/173168

Temerin, M., Roth, I.: The production of ${ }^{3} \mathrm{He}$ and heavy ion enrichment in ${ }^{3} \mathrm{He}$-rich flares by electromagnetic hydrogen cyclotron waves. Astrophys. J. Lett. 391, L105 (1992). https://doi. org/10.1086/186408

Vršnak, B., Cliver, E.W.: Origin of coronal shock waves. Sol. Phys. 253, 215 (2008). https://doi. org/10.1007/s11207-008-9241-5

Wang, Y.-M., Pick, M., Mason, G.M.: Coronal holes, jets, and the origin of ${ }^{3} \mathrm{He}-$ rich particle events. Astrophys. J. 639, 495 (2006). https://doi.org/10.1086/499355

Yashiro, S., Gopalswamy, N., Cliver, E.W., Reames, D.V., Kaiser, M., Howard, R.: Association of coronal mass ejections and type II radio bursts with impulsive solar energetic particle event. In: Sakurai, T., Sekii, T. (eds.) The Solar-B Mission and the Forefront of Solar Physics, ASP Conf. Ser. 325, 401 (2004)

Open Access This chapter is licensed under the terms of the Creative Commons Attribution 4.0 International License (http://creativecommons.org/licenses/by/4.0/), which permits use, sharing, adaptation, distribution and reproduction in any medium or format, as long as you give appropriate credit to the original author(s) and the source, provide a link to the Creative Commons license and indicate if changes were made.

The images or other third party material in this chapter are included in the chapter's Creative Commons license, unless indicated otherwise in a credit line to the material. If material is not included in the chapter's Creative Commons license and your intended use is not permitted by statutory regulation or exceeds the permitted use, you will need to obtain permission directly from the copyright holder.

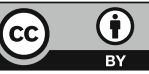

